\title{
Efecto de un CD multimedia (planeta riesgo xero) en los conocimientos, actitudes y prácticas sobre enfermedades de transmisión sexual y VIH/sida de adolescentes de colegios de Lima Metropolitana
}

\author{
Aníbal Velásquez ${ }^{1}$
}

Resumen

Palabras clave

\begin{abstract}
Objetivos: Determinar el efecto del CD Planeta Riesgo Xero en los conocimientos actitudes y prácticas relacionadas a las ITS y VIH/sida de adolescentes de Colegios de Lima. Materiales y Métodos: El estudio se realizó en una muestra aleatoria de los alumnos del cuarto y quinto año de secundaria de 26 colegios estatales y particulares. Participaron del estudio 454 adolescentes de 14 a 17 años, a quienes se les aplicó una encuesta de "Conocimientos, Actitudes y Prácticas” antes y después de la exposición al CD educativo. Resultados: El 22\% de adolescentes refirió haber tenido relaciones sexuales (coitales) y alrededor de 8\% sufrió algún tipo de abuso sexual. La comparación pre y post intervención de conocimientos, actitudes y prácticas muestra diferencias significativas. Se logró incrementar en más de $20 \%$ los conocimientos sobre ITS y el VIH/sida, en $21 \%$ los conocimientos sobre las formas de transmisión y en $16 \%$ sobre las formas de prevención. Después de la exposición al CD educativo, 9\% más adolescentes piensan usar el condón en sus relaciones sexuales. Conclusiones:El CD Planeta Riesgo Xero ha sido reconocido por los adolescentes como un medio entretenido y útil para aprender cómo prevenir las enfermedades de transmisión sexual y el VIH/Sida. Los resultados de este estudio demuestran que este medio educativo mejora los conocimientos de los adolescentes en esta materia.
\end{abstract}

Materiales de enseñanza; medios audiovisuales; enfermedades sexualmente transmisibles; adolescencia; síndrome de inmunodeficiencia adquirida; infecciones por VIH.
Effect of a multimedia CD (Xero Risk Planet) on Metropolitan Lima school teenagers knowledge, attitudes and practices on sexually transmitted diseases and HIV/AIDS

Abstract

Objectives: To determine the effect of the CD Xero Risk Planet on Lima school teenagers knowledge, attitudes and practices on relation to STD and HIV/AIDS. Materials and Methods: The study was done in a random sample of fourth and fifth grade high school students from 26 schools. A questionnaire of knowledge, attitudes and practices was

1 MD, MPH, Profesor de la Universidad Nacional Mayor de San Marcos, Posgrado de Economía de la Salud. Consultor en evaluación de programas sociales y de salud. Lima, Perú. applied to 454 teenagers (14 to 17 years old), before and after the use of the educational CD. Results: Twenty-two percent of interviewed teenagers had had sex, about $8 \%$ had undergone some type of sexual abuse. Knowledge on STD and HIV/AIDS transmission and prevention increased (20\%, 21\% and $16 \%$, respectively) following the intervention. Also, 9\% more teenagers think to use condom during their sexual relations. Conclusions: The Xero Risk Planet CD has been acknowledged by young people as a useful and entertaining way of learning on STDs and HIV/ AIDS prevention. The results of the study demonstrate that this educational tool significantly improved young peoples' knowledge on the subject.

Keywords: Teaching materials; audiovisual aids; sexually transmitted diseases; adolescence; acquired immunodeficiency syndrome; HIV infections. 


\section{INTRODUCCIÓN}

La sexualidad del adolescente es un tema tabú, por lo que las estrategias educativas dirigidas a este grupo de riesgo ha generado diversas críticas de los padres y de algunos sectores de la sociedad. Cuando se ha pretendido educar abiertamente sobre la salud sexual al adolescente, algunos lo han calificado como un atentado contra la moral, aludiendo que se está promoviendo la sexualidad precoz. Sin embargo, la sexualidad precoz y las relaciones sexuales premaritales existen en nuestra sociedad, a pesar de no ser aceptado socialmente.

Los estudios nacionales nos indican que las relaciones sexuales premaritales, son cada vez más precoces, más frecuentes y con múltiples parejas, lo que se refleja en las altas tasas de embarazos en adolescentes, embarazos no deseados y abortos en mujeres jóvenes, así como altas tasas de infecciones de transmisión sexual y sida. Los adolescentes son más susceptibles conductual y biológicamente a tener relaciones sexuales y están más expuestos a la coerción sexual económica, familiar y cultural, siendo mayor si se usa alcohol y drogas.

En Latinoamérica, los jóvenes inician su actividad sexual a la edad de 12,7 a 16 años, mientras que las mujeres lo hacen a la edad de 15,6 a 17,9 años $\left(^{1}\right)$. La incidencia de las infecciones de transmisión sexual (ITS) en jóvenes se ha incrementado dramáticamente en los últimos 20 años. Cerca de la mitad de los infectados por virus de inmunodeficiencia humana (VIH) actualmente son menores de 25 años. La incidencia de sida se está incrementando rápidamente en mujeres jóvenes. En el Perú, 3\% de los casos de sida se presenta en el grupo etáreo de 15 a 19 años $\left({ }^{2}\right)$.

Los jóvenes tienen alto riesgo para las ITS, debido principalmente a sus conductas. Ellos son vulnerables si no usan condones consistente y correctamente. La mayoría de jóvenes tiene poco conocimiento de las ITS y sus síntomas y muchos no reciben tratamiento oportuno. En una encuesta realizada por Inppares en una muestra representativa de colegios de Lima Metropolitana, más de $30 \%$ de los adolescentes entrevistados no conocía sobre las enfermedades de transmisión sexual, más de $20 \%$ no sabía que el condón puede prevenir enfermedades de transmisión sexual y VIH/sida, $40 \%$ no sabía cómo se propaga el sida y sólo $21 \%$ refería que utilizaba condón en sus relaciones sexuales $\left({ }^{3}\right)$. Según la Encuesta Nacional Demográfica y de Salud Familiar (ENDES 1996), $21 \%$ de las mujeres de 15 a 19 años no conoce alguna ITS.

La gente joven que inicia sus relaciones sexuales más temprano tienen más probabilidades de tener más de una pareja; por tanto, se incrementa la posibilidad de adquirir una ITS. Biológicamente, las mujeres jóvenes tienen mayor susceptibilidad a infecciones de transmisión sexual debido a ectopia de cérvix. Ésta es una condición normal que se presenta en adolescentes y que llega a ser menos común con la edad; las células endocervicales son más vulnerables a las infecciones, como la clamidia y gonorrea.

Frente a estos hechos, se ha propuesto intervenciones sobre la conducta para la prevención de enfermedades transmitidas sexualmente; $y$, en los años 80 , se comenzó programas para prevenir las infecciones por el virus del sida. Aral y Peterman $\left({ }^{4}\right)$ realizaron una revisión sobre la efectividad de intervenciones conductuales para prevenir las ITS y la infección por VIH y concluyeron que estos estudios no han podido demostrar cambios conductuales.

Se ha probado que las clases tradicionales muestran tasas bajas de asimilación, a diferencia de la mayor cantidad de información y mayor velocidad de asimilación que la generación de jóvenes tienen cuando utilizan juegos electrónicos y educativos multimedia $\left({ }^{5}\right)$. De esta forma, la producción y uso de sistemas multimedia representa un poderoso asistente para la educación en diferentes áreas. Los modelos hipertextohipermedia representan de manera más natural, y no lineal, la estructuración del conocimiento $\left({ }^{6}\right)$.

De acuerdo a Struchiner $\left(^{7}\right)$, la importancia de los sistemas multimedia en educación se caracteriza por: a) la interactividad permite la independencia del estudiante en la elección de la información y en el ritmo de trabajo; b) la integración de diversas vías, porque se presenta la información en diferentes formas; en consecuencia, permite 
diferentes estilos y preferencias de aprendizaje; c) no existe linealidad de la información, los estudiantes utilizan mecanismos de asociaciones de información (lo que es una manera natural de aprender). En general, estos sistemas confieren al estudiante autonomía y responsabilidad en su propio proceso de aprendizaje, porque privilegian la interacción, los intereses del alumno, el descubrimiento y los ritmos de aprendizaje $\left({ }^{8}\right)$; esto le confiere al profesor un rol de facilitador, creador de ambientes de aprendizaje, en vez de ser un simple transferidor de conocimiento.

Por lo expuesto, se propuso desarrollar un programa educativo en multimedia, denominado Planeta Riesgo Xero, con el fin de incrementar los conocimientos sobre las ITS y el VIH/sida de los adolescentes y promover conductas que posterguen el inicio de las relaciones sexuales y las prácticas de sexo seguro. Se promueve de manera interactiva y entretenida la reflexión de los adolescentes sobre las conductas y situaciones de riesgo a las que está expuesto, utilizando 1) seis historias cotidianas de adolescentes; 2) mensajes sobre autoestima, asertividad, valores, toma de decisiones y comunicación con los padres; 3 ) un juego sobre toma de decisiones para evitar ITS y VIH/sida; 4) Una prueba para medir el riesgo; 5) Información sobre ITS a partir de los síntomas y signos de mujeres y hombres; 6) Información sobre VIH/sida; y, 7) Mitos y creencias.

El proyecto se evaluó con un cuestionario de Conocimientos, Actitudes y Prácticas antes y después de realizar la intervención con el CD. Participaron del estudio 454 estudiantes de cuarto y quinto de secundaria de 26 colegios de Lima, cuyos resultados se presenta en este estudio

El objetivo de la presente investigación fue determinar los cambios de conocimientos y actitudes de los adolescentes acerca de las ITS y VIH/sida después de utilizar el CD educativo multimedia Planeta Riesgo Xero.

\section{MATERIALES Y MÉTODOS}

La evaluación de la intervención educativa en los colegios se realizó con un diseño preexperimental, para lo cual se aplicó una encuesta de conocimientos, actitudes y prácticas para prevenir ITS y VIH/sida antes y después de utilizar el educativo multimedia "Planeta Riesgo Xero" a adolescentes de colegios de Lima Metropolitana.

La población del estudio fue los adolescentes de ambos sexos que se encontraban cursando cuarto y quinto año de educación secundaria en colegios de Lima Metropolitana. Participaron 26 colegios: 5 estatales, 17 particulares, 3 de las fuerzas armadas y un colegio parroquial.

La unidad de muestreo fue los alumnos de los colegios participantes de la investigación; el marco muestral utilizado para determinar el tamaño de muestra y la selección de los alumnos fue el listado de todos los alumnos de cuarto y quinto de secundaria de los colegios participantes.

El diseño muestral utilizado fue probabilístico, bietápico: unidad primaria de muestreo (colegio), unidad secundaria de muestreo (alumno). En la primera etapa se utilizó un muestreo autorrepresentado de colegios. En la segunda etapa, se usó la relación de asistencia de los alumnos de cuarto y quinto, se seleccionó a los alumnos por medio de muestreo sistemático aleatorio.

El tamaño muestral calculado fue 454 , con un margen de error de $4,6 \%$, un nivel de confianza del $95 \%$ y con una tasa de no-respuesta del $10 \%$, cuya utilización se debe a experiencias de estudios anteriores llevados a cabo por otras organizaciones, tratándose de un tema sensible como es la sexualidad en los adolescentes; el tamaño de la muestra se distribuyó en 26 colegios.

Luego de un mes de intervención, se encuestó a los mismos alumnos que fueron entrevistados antes de utilizar el CD 'Planeta Riesgo Xero'.

El cuestionario tuvo 101 preguntas en cuatro secciones: Sección I (datos generales) comprendió datos generales del entrevistado; Sección II (comportamiento y conducta), comunicación con la pareja sobre temas relacionados con sexualidad, tipo de compañero sexual, edad en la primera relación sexual y cuál fue la razón que lo(a) llevó a tener su primer contacto sexual; Sección III (conocimientos - ITS), los conocimientos de los 
adolescentes acerca de las infecciones de transmisión sexual, ¿cuáles son las enfermedades que ellos conocen?, las formas de contagio, consecuencias y sobre las formas de prevención; Sección IV (sida) contenía preguntas relativas al tema, como ¿Qué es sida?, ¿Qué significa sida?, las formas de transmisión de la enfermedad, formas de prevención y ¿Cuáles son los síntomas del sida?

El cuestionario fue validado en una prueba piloto con alumnos de cuarto y quinto de secundaria, con cuyo resultado fue reajustado.

El programa educativo multimedia ha sido desarrollado en el Instituto Peruano de Paternidad Responsable (Inppares). El nombre, los guiones, la estructura del programa, los colores, la música y los personajes han sido desarrollados por expertos en el manejo de software multimedia (Director, Photoshop, Illustrator, Adobe Premier) y por consultores que trabajan con jóvenes del Procetts, Centro de Jóvenes Futuro de Inppares, International Planned Parenthood (IPPF); fueron validados por adolescentes y profesores de los colegios participantes.

Planeta Riesgo Xero es un educativo multimedia que informa y educa de manera entretenida sobre la prevención de infecciones de transmisión sexual y el virus del VIH/sida, informándole sobre los riesgos a los que están expuestos los adolescentes. El programa educativo se basa en una historia de dos adolescentes cibernéticos de un planeta llamado Riesgo XERO, los mismos que recorren junto con el usuario por el espacio gráfico y virtual del programa en forma interactiva.

El programa contiene seis videos con historias de jóvenes que están expuestos a infecciones de transmisión sexual y al virus del sida; en tres de los videos el usuario puede elegir el desenlace final, dependiendo de la elección que realice el usuario.

Al final de cada historia, se presenta mensajes que refuerzan la autoestima, asertividad, y adecuada toma de decisiones del adolescente. Estos mensajes promueven la comunicación con los padres y la formación de valores.

Planeta Riesgo Xero cuenta también con una prueba para evaluar el riesgo de adquirir ITS y
VIH/sida de los usuarios del programa. Contiene además un juego que promueve y enseña a tomar decisiones para evitar estas enfermedades, introduciendo el concepto de que la probabilidad de adquirirlas se incrementa cuando no se toma buenas decisiones.

La información sobre las ITS se enseñó a partir de los síntomas de las enfermedades, tanto para hombres como mujeres.

Finalmente, incluye un módulo sobre el correcto uso del condón, creencias y prejuicios sobre el condón y el sida y consejos y direcciones a dónde se pueden dirigir los adolescentes en caso lo requieran.

Las encuestas estuvieron a cargo de tres personas con experiencia en esta labor y con nivel de educación superior (psicología). Los encuestadores fueron capacitados en cuanto a manejo de grupo, en el manejo de cuestionario y objetivos de la investigación. Se les proporcionó un Manual del Entrevistador para el trabajo de campo, en el cual se mencionaba los objetivos de la investigación, descripción del cuestionario, técnica de selección de alumnos, tareas y responsabilidades del encuestador y supuestos de posibles problemas a presentarse y formas de solucionarlo.

El procesamiento de la información se realizó en EPI INFO 6,04. Previamente, se diseñó la base de datos con la estructura del cuestionario comprendiendo todo el rango de códigos numéricos correspondiente a las variables y filtros necesarios, a fin de evitar errores y facilitar el ingreso de datos.

La base de datos fue revisada (consistencia de la base de datos) para detectar los posibles errores al ingresar los datos. El análisis final se realizó en SPSS.

Para medir el grado de diferencia de conocimientos antes de la aplicación del CD y luego de la intervención, se utilizó la prueba de McNemar (utilizado para diseños pareados y con variables categóricas dicotómicas).

Se contrastó la siguiente hipótesis nula: Hipótesis nula(Ho): No existe cambio con relación a los conocimientos y actitudes sobre ITS y VIH/ sida de los alumnos seleccionados entre antes y 
después de haberse implementado el CD "Planeta Riesgo Xero".

\section{RESULTADOS}

Los adolescentes entrevistados tenían entre 14 a 17 años de edad. Cerca de tres cuartos $(74,4 \%)$ correspondió a familia nuclear, es decir, conformados por padre, madre y hermanos. El 50\% deseaba seguir estudios superiores, seguidos por aquellos que pensaban combinar el trabajo con el estudio (39\%).

Respecto a las relaciones familiares, sólo 50\% de los adolescentes entrevistados manifestó que las relaciones familiares eran buenas. Más de $40 \%$ manifestó ser víctima de algún tipo de maltrato psicológico y/o físico en el seno familiar.

El 22\% de adolescentes refirió haber tenido relaciones sexuales (coitales), la mayoría las inició entre los 13 y 15 años (67\%). El 34\% de las mujeres refirió que inició sus relaciones sexuales antes de los 16 años de edad. La razón más frecuente para que se iniciara sexualmente la mujer fue por amor $(61,5 \%)$ y $46 \%$ de los varones manifestó que se les presentó la oportunidad. Se encontró que los adolescentes inician generalmente su actividad sexual en su entorno más próximo, principalmente con enamorado $(85 \%$ de las mujeres y $51 \%$ de los hombres).

La primera información sobre sexualidad fue brindada en mayor porcentaje por profesores $(37,2 \%)$ y sus padres $(36,8 \%)$. Estos porcentajes cambiaron cuando se trataba de las personas a quienes recurrían los adolescentes cuando necesitan información sobre sexualidad; los adolescentes mencionaron que recurrían en primer lugar a los amigos $(70 \%)$.

El estudiante se identificó generalmente con alguien de su mismo sexo para discutir temas relacionados a la sexualidad. Es decir, $48 \%$ de los hombres respondió que consultó a su padre y $68 \%$ de las mujeres afirmó que prefería consultar o hablar con su madre sobre sexualidad.

Alrededor de $8 \%$ de los adolescentes entrevistados manifestó haber sufrido algún tipo de abuso sexual, siendo mayor en las mujeres $(13,3 \%)$ y $4 \%$ en el caso de varones.
La prostitución es otro tema que afectó a los adolescentes, ya que alrededor de $13 \%$ de los hombres y $7 \%$ de mujeres fueron tentados a tener relaciones sexuales a cambio de dinero, regalos o favores. Alrededor del 2\% de los hombres dijo haber aceptado tal proposición, en el último año.

El $13,5 \%$ de los adolescentes entrevistados utilizó condón y $4 \%$ píldoras anticonceptivas. El $46 \%$ de las mujeres y $58 \%$ de los varones refirió que utilizó condón como método en la primera relación sexual. Y 31\% de mujeres y 38\% de varones afirmaron que los usan en todas las relaciones sexuales.

Antes de la exposición al CD educativo, 30\% de los entrevistados reconocía las ITS y después del uso del CD este porcentaje se incrementó a $49 \%$. Se observó un incremento significativo de $19 \%(p<0,001)$. Luego de utilizar el CD, los adolescentes tuvieron 3,5 veces más probabilidad de reconocer una ITS en su pareja.

El conocimiento de los síntomas de las ITS que mostraron diferencias significativas al comparar las respuestas de antes y después del uso del CD, fueron: picazón en los genitales (16\% de diferencia, $p=0,035$ ), presencia de pus en el pene (18\% de diferencia, $p=0,019)$. Los adolescentes que utilizaron el CD tuvieron cerca de tres veces más probabilidades de asociar la picazón de los genitales a una ITS y casi 4 veces más de asociar pus en el pene con una ITS (Tabla 1).

En la Tabla 1 también se muestra los cambios de los conocimientos de los adolescentes entrevistados en cuanto a los tipos de ITS: gonorrea, sífilis, Chlamydia, herpes genital y chancro blando. Los adolescentes conocían menos la Chlamydia $(2 \%)$ y el chancro blando $(36 \%)$ antes de utilizar el CD. El incremento de adolescentes que respondieron conocer las ITS fue para gonorrea $26 \%$ más $(p<0,001)$, para sífilis $24 \%$ más $(p<0,001)$, para clamidia $22 \%$ más $(p<0,001)$, herpes genital $21 \%$ más $(p<0,001)$ y el chancro blando $13 \%$ más $(p<0,001)$. La probabilidad de que después del uso del CD conocieran la gonorrea fue 5 veces más, para sífilis 5 veces más, clamidia 13 veces más, herpes genital 4 veces más y para el chancro blando 2 veces más. 
También se incrementó el porcentaje de adolescentes que conocían el VIH (15\% más, $p<0,001)$ y el sida $(17 \%$ más, $p<0,016)$. Los adolescentes tuvieron 3 veces más probabilidad de conocer el VIH y 6 veces más de conocer el sida luego de estar expuestos al educativo multimedia. Así mismo, más adolescentes (23\% más) conocieron que las relaciones sexuales orales también pueden ser formas de transmisión del VIH $(\mathrm{OR}=5,7 ; p<0,001)$ y que el VIH se transmite también por tener relaciones sexuales anales $(18 \%$ más, $\mathrm{OR}=3,9 ; p<0,001)$.

Del mismo modo, se incrementaron los conocimientos sobre las formas de prevención. Más adolescentes conocieron que son formas de prevención la abstinencia sexual (11\% más, $p<0,001)$, el retraso del inicio de las relaciones sexuales $(27 \%$ más, $p<0,001)$ y evitando compartir objetos personales (11\% más, $p<0,001)$.

Las actitudes y prácticas que mostraron algún cambio significativo fueron las preguntas referidas a situaciones de riesgo de adquirir una ITS o la infección con el VIH. En la mayoría de las respuestas después de utilizar el CD "Planeta Riesgo Xero" se mostró un incremento de adolescentes con actitudes favorables para prevenir estas infecciones.

Con relación al uso de tatuajes, las opiniones cambiaron luego de utilizar el CD, $8 \%$ menos respondieron que se realizarían un tatuaje $(p=0,002)$. Se encontró que utilizar el CD ofrece aproximadamente dos veces más de probabilidades de que el adolescente no se realice tatuajes (Tabla 2). Del mismo modo se reduce en 3 veces la creencia de que a mayor número de parejas sexuales se es más popular y se redujo 10 veces la creencia de que para desarrollarse mejor se necesita tener relaciones sexuales.

Luego de utilizar el CD educativo, menos adolescentes respondieron que "las personas que llevan condones consigo tienen más sexo con gente" ( $15 \%$ menos, $p<0,001)$, aumentando la probabilidad de no tener esta creencia luego de utilizar el CD de aproximadamente 5 veces más (Tabla 2).

Tabla 1. Cambios en los conocimientos para reconocer ITS y VIH/sida de los adolescentes de Colegios de Lima Metropolitana que utilizaron el CD educativo "Planeta Riesgo Xero"

\begin{tabular}{|c|c|c|c|c|c|}
\hline \multirow[t]{2}{*}{ Variable } & \multicolumn{3}{|c|}{ Cambios logrados } & \multirow[t]{2}{*}{$p$} & \multirow[t]{2}{*}{ OR } \\
\hline & $\begin{array}{c}\text { Antes } \\
\%\end{array}$ & $\begin{array}{c}\text { Después } \\
\%\end{array}$ & $\begin{array}{c}\text { Cambio } \\
\%\end{array}$ & & \\
\hline \multicolumn{6}{|l|}{ Reconocimiento de ITS } \\
\hline Pueden reconocer la presencia de alguna ITS en su pareja sexual & 30 & 49 & 19 & $<0,001$ & 3,5 \\
\hline Conocimiento de síntomas de ITS: “Picazón en los genitales” & 18,8 & 34,5 & 15,7 & 0,035 & 2,8 \\
\hline \multicolumn{5}{|l|}{ Agentes causales de ITS } & 3,8 \\
\hline Reconoce la gonorrea & 45,2 & 71,5 & 26,3 & $<0,001$ & 5,4 \\
\hline Reconocer la sífilis & 45,8 & 69,7 & 23,9 & $<0,001$ & 4,8 \\
\hline Reconocer la Chlamydia & 2,1 & 24,2 & 22,1 & $<0,001$ & 13,2 \\
\hline Reconocer el herpes genital & 52,7 & 73,9 & 21,2 & $<0,001$ & 3,7 \\
\hline Reconocer el chancro blando & 35,8 & 48,8 & 13 & $<0,001$ & 2,3 \\
\hline \multicolumn{6}{|l|}{ Conocimiento sobre VIH/sida } \\
\hline Conocer qué es el VIH & 38,5 & 53,9 & 15,4 & $<0,001$ & 3,5 \\
\hline Sabe qué es el sida & 43,6 & 60,3 & 16,7 & 0,016 & 6,0 \\
\hline \multicolumn{6}{|l|}{ Formas de transmisión del VIH/sida } \\
\hline Relaciones sexuales orales & 38,8 & 62,4 & 23,6 & $<0,001$ & 5,7 \\
\hline Relaciones sexuales anales & 59,1 & 77,3 & 18,2 & $<0,001$ & 3,9 \\
\hline \multicolumn{6}{|l|}{ Formas de prevención } \\
\hline Abstinencia sexual & 66,1 & 76,7 & 10,6 & $<0,001$ & 2,7 \\
\hline Retrazar el inicio de las relaciones sexuales & 25,2 & 52,4 & 27,2 & $<0,001$ & 5,2 \\
\hline No compartir objetos personales & 81,5 & 92,4 & 10,9 & $<0,001$ & 4,0 \\
\hline
\end{tabular}


Tabla 2. Cambios en las actitudes y las prácticas sobre prevención de ITS y VIH/sida.

\begin{tabular}{|c|c|c|c|c|c|}
\hline \multirow[t]{2}{*}{ Variable } & \multicolumn{3}{|c|}{ Cambios logrados } & \multirow[t]{2}{*}{$p$} & \multirow[t]{2}{*}{ OR } \\
\hline & $\begin{array}{c}\text { Antes } \\
\%\end{array}$ & $\begin{array}{c}\text { Después } \\
\%\end{array}$ & $\begin{array}{l}\text { Cambio } \\
\%\end{array}$ & & \\
\hline \multicolumn{6}{|l|}{ Sobre prevención de ITS y VIH/sida } \\
\hline Tienes o te gustaría hacerte algún tatuaje & 45,5 & 37,6 & $-7,9$ & 0,002 & 0,43 \\
\hline A mayor parejas sexuales mayor popularidad & 18,2 & 10,3 & $-7,9$ & $<0,001$ & 0,3 \\
\hline $\begin{array}{l}\text { Para ser más maduro y desarrollarse bien los } \\
\text { hombres y mujeres deben tener relaciones sexuales }\end{array}$ & 5,8 & 0,6 & $-5,2$ & $<0,001$ & 0,1 \\
\hline Sobre el uso del condón & & & & & \\
\hline $\begin{array}{l}\text { Las personas que cargan condones tienen } \\
\text { sexo con más gente }\end{array}$ & 32,1 & 17,6 & $-14,5$ & $<0,001$ & 0,22 \\
\hline Usarías condón o le pedirías a tu pareja que los use & 89,7 & 96,7 & 7 & $<0,001$ & 7,33 \\
\hline $\begin{array}{l}\text { El aprender más acerca de las ITS cambió el } \\
\text { comportamiento en: usar condón en toda situación }\end{array}$ & 38,8 & 47,9 & 9,1 & 0,006 & 1,76 \\
\hline
\end{tabular}

Se incrementó en $7 \%$ la opinión de que los adolescentes pedirían a sus parejas que utilicen condón $(p<0,001, \mathrm{OR}=7)$, en $9 \%$ el uso del condón en toda ocasión $(p=0,006, \mathrm{OR}=2)$.

El incremento de adolescentes que respondieron que estarían de acuerdo en ser fieles a sus parejas sólo se incrementó en $3 \%$, considerando que desde el inicio esta respuesta ha sido alta $(90 \%$ de los adolescentes estuvieron de acuerdo antes del uso del CD). De todas formas, se ha encontrado diferencia significativa entre la comparación de las respuestas antes y después $(p=0,038)$.

\section{DISCUSIÓN}

La propuesta de utilizar una estrategia educativa en multimedia en los colegios se debe a que los grupos de edades con mayor vulnerabilidad de infección de ITS y del VIH pasan la mayor parte de su tiempo en los colegios; por esta razón, programas educativos de prevención podrían ayudar a prevenir conductas de riesgo. En los Estados Unidos, esta estrategia ha demostrado resultados favorables en el incremento del uso de condones y de las conductas preventivas en los jóvenes $\left({ }^{9}\right)$. El programa educativo de los colegios no incluye la educación de conductas preventivas, por lo que una estrategia complementaria entretenida y con información personalizada e interactiva tendría un efecto positivo en la disminución de las infecciones.
En el presente estudio no es posible medir el impacto en la incidencia de estas enfermedades. El objeto del estudio es mostrar cambios en los conocimientos, en actitudes y prácticas que son el inicio del desarrollo de conductas preventivas. Las intervenciones sobre la conducta para la prevención de enfermedades transmitidas sexualmente comenzaron con mayor intensidad en los años 80, de manera de prevenir las infecciones por el virus del sida, principalmente.

Sobre la efectividad de las intervenciones conductuales para prevenir las ITS y la infección por VIH, se ha mostrado el efecto sobre los conocimientos, actitudes, percepciones y habilidades sobre el uso de condones, el número de parejas sexuales y la práctica de sexo no protegido $\left({ }^{4}\right)$.

En este estudio se investigan los cambios en los conocimientos, actitudes y prácticas sobre las ITS y el VIH/sida relacionados a la exposición de adolescentes de colegios a un CD ROM educativo multimedia denominado "Planeta Riesgo Xero". Los cambios en las actitudes y prácticas encontrados no necesariamente pueden ser atribuidos al efecto del CD, debido a que el diseño utilizado (preexperimental) no permite controlar todas las variables confusoras, por lo que sólo podemos hablar de asociación entre el cambio encontrado y el programa educativo multimedia. Un diseño experimental controlado y aleatorizado es factible y apropiado en este caso $\left({ }^{10}\right)$. 
Luego de la exposición al CD educativo, fueron más marcados los cambios en los conocimientos que en las actitudes y prácticas. Este hallazgo coincide con otros estudios que prueban estrategias educativas para modificar las conductas de riesgo de infecciones de transmisión sexual y del VIH. Los cambios en las prácticas no fueron medidos directamente en esta investigación; sólo se pudo medir la intención de tener determinadas prácticas.

Se destaca que los adolescentes hayan referido un cambio significativo en los conocimientos sobre dos de los síntomas de las ITS, el prurito y pus por el pene. La razón se puede deber a que el descenso de flujo vaginal fue más conocido por los adolescentes entrevistados y la diferencia no fue notoria después de utilizar el CD ROM. Los otros síntomas que se enseña en el CD tal vez requieren de mayor tiempo de uso del educativo multimedia u otra forma más efectiva de transmitir este conocimiento, dado que los grupos sindrómicos más vistos fueron úlcera genital y flujo vaginal. Los efectos del CD ROM fueron mayores cuando los conocimientos sobre algún ítem eran más escasos, como la Chlamydia.

Llama la atención de que, a pesar de que se encuentre difundido el tema del VIH y el sida, aún hay estudiantes que no lo conocen. Este hallazgo es similar al encontrado en un estudio realizado por el PROCETSS en el Cono Sur de Lima $\left({ }^{11}\right)$.

Se destaca que con el educativo multimedia se incrementó el conocimiento de que son formas de transmisión del VIH las relaciones sexuales orales $\mathrm{y}$ anales.

Con el educativo multimedia más adolescentes respondieron que el retraso de las relaciones sexuales es una forma de prevención de las ITS y del VIH /sida. Se atribuye que el efecto fue mayor debido al bajo nivel de conocimientos que existía antes de la intervención.

Las actitudes y prácticas que mostraron algún cambio significativo fueron las preguntas referidas a las situaciones de riesgo de adquirir ITS o la infección del VIH. Se encontró cambios de percepción de riesgo sobre los tatuajes y tener más parejas sexuales.

El incremento de los deseos de utilizar condón en las relaciones sexuales no se ha incrementado notoriamente como los conocimientos, debido probablemente a que muchos de los adolescentes entrevistados no tienen relaciones sexuales aún. Se debe anotar que el CD promueve el uso de condón en toda relación sexual y que las historias más vistas fueron: "Mi Gran Amor" y "Si me quieres demuéstralo" (historias sobre el uso del condón); sin embargo, el efecto no ha sido mayor que lo esperado en temas relacionados al uso del condón.

La fidelidad, como una forma de prevenir ITS y la infección por el virus del sida, ha sido una respuesta frecuente antes de que se intervenga con este programa educativo, hallazgo que es similar al del estudio del Cono Sur de Lima $\left({ }^{11}\right)$ y de Angela Sebastiani y col $\left({ }^{3}\right)$.

Los cambios de conocimientos y actitudes mostrados en el estudio apoyan la hipótesis de que esta forma de aprender es efectiva por no ser lineal $\left({ }^{6}\right)$, debido a que la educación en sistemas multimedia utiliza mas bien mapas conceptuales $\left.{ }^{(12}\right)$. Los mapas conceptuales son una forma gráfica de representación del conocimiento en el cual toda la información importante puede ser encajada en nodos y arcos (líneas que conectan nodos). Esta forma de aprender se caracteriza por ser flexible y se supone que la flexibilidad del educativo multimedia motiva el aprendizaje sobre el tema.

En el CD multimedia hay una estructura jerárquica implícita del conocimiento. Toda la información está representada en textos, gráficos y vídeos, que puede ser accedida por diversas vías. La información en texto siempre es presentada en forma breve, compacta y clara. En esencia, el CD "Planeta Riesgo Xero" es un mapa de ruta hacia el conocimiento de las ITS y del VIH/sida.

Otra explicación del efecto del CD "Planeta Riesgo Xero" es la metodología constructivista basada en problemas y en el pensamiento crítico con el que fue diseñado. Esta forma de diseñar educativos multimedia ha sido descrita por Albion y Gibson $\left({ }^{13}\right)$. La teoría constructivista parte de que la comprensión está conectada a la interacción entre el aprendizaje y el ambiente $\left({ }^{14}\right)$, que el conflicto cognoscitivo o los rompecabezas son los estímulos para el aprendizaje. Esta metodología ha sido introducida en la educación médica a mediados de los años 60 con éxito $\left({ }^{15}\right)$. 
Se puede afirmar que la autonomía y responsabilidad en su propio proceso de aprendizaje que ejerce el estudiante utilizando el multimedia educativo "Planeta Riesgo Xero" favorecieron el aprendizaje sobre las ITS y el VIH/ sida. Los sistemas multimedia privilegian la interacción, los intereses del alumno, el descubrimiento y los ritmos de aprendizaje ${ }^{8}$ ).

Podemos concluir que el CD ROM "Planeta Riesgo Xero" ha demostrado una asociación con el cambio de conocimientos sobre las infecciones de transmisión sexual y el VIH/sida y, en menor proporción, con el cambio de actitudes y de prácticas.

Los alumnos calificaron de muy bueno el CD ROM y que les hizo reflexionar sobre los riesgos de contraer estas enfermedades. Así mismo, indicaron que la información era más que buena.

Las herramientas multimedia pueden ser utilizadas con éxito en los colegios para generar cambios de conducta favorables a prevenir estas enfermedades; para esto es necesario que los profesores promuevan el uso de educativos multimedia.

\section{AGRADECIMIENTOS}

Al Instituto Peruano de Paternidad Responsable - Inppares-, a International Planned Parenthood Federation -IPPF-Trust Fund, por haber financiado el desarrollo del CD educativo y la investigación. A Betty Manrique y Martha Díaz por el apoyo en el análisis estadístico.

\section{REFERENCIAS BIBLIOGRÁFICAS}

1. Moya C. The sexual and reproductive health of young people in Latin America and the Caribbean [monografía en la Internet]. Washington, DC: Advocates for youth; 2002 [citado 2005-0804]; Disponible en: http://www.advocatesforyouth.org/ publications/factsheet/fssexualhealth.htm

2. Ministerio de Salud del Perú, Ofícina General de Epidemiología. Situación del VIH/SIDA en el Perú [monografía en la Internet]. Lima: Ministerio de Salud; 2003 [citado 2005-08-04]. Disponible en: http:// www.impactaperu.org/download/pdf0340.pdf

3. Sebastiani A, Segil E. ¿Qué hacen?, ¿qué piensan?, ¿qué sienten? los y las adolescentes de Lima respecto a la salud sexual y reproductiva. $1^{\text {ra }}$ Ed. Lima: Inppares; 1998.
4. Aral SO, Peterman TA. Do we know the effectiveness of behavioral interventions? The Lancet. 1998;351(Suppl III):33-6.

5. Naegeli C, Antonini R, Ellwagner G. Development of didactic aids engineering education: multimedia on strength of materials and structures behavior [monografía en la Internet]. Rio de Janeiro: ICEE; 1998 [citado 2005 Ago 4]. Disponible en: http://www.ctc.puc-rio.br/icee-98/Icee/ papers/460.pdf

6. Cimino JJ, Elkin PL, Bamet GO. As we my think: the concept space and medical hypertext. Computers and Biomedical Research. 1992;25(3):238-63.

7. Struchiner M. Hiper Text and Hyper Media in the Education, Basic Principles for the Development of Educative Material. Río de Janeiro: Casa da Ciencia/UFRJ; 1996.

8. Landow G. Hypertext. The convergence of contemporary critical theory and technology [monografía en la Internet]. Baltimore: Johns Hopkins University Press; 1995 [citado 2005-08-04]. Disponible en: http://www.cyberartsweb.org/ cpace/ht/jhup/contents.html

9. Control Disease Control. School-based HIV-prevention education- United States, 1994. MMWR. 1996;45(35):760-5.

10. Stephenson J, Oakley A, Charleston S, Brodala A, Kenton $\mathrm{K}$, Petruckevitch, et al. Behavioral interventions trials for HIV/STD prevention in schools: are they feasible? Sexually Transmitted Infections. 1998;74:405-8.

11. PROCETSS, Calandria. Los Tromes del Cono Sur. Lima: Contrasida Informa MINSA, 1999

12. Kopec D. Introduction to smartbooks. AIDS II Smartbook version [monografía en la Internet]. Brooklyn: University of New York; 1993 [citado 2005-08-04]. Disponible en: http://spider.sci.brooklyn.cuny.edu/ kopec/Publications/ AIDSII Brochure.pdf

13. Albion P, Gibson I. Designing multimedia materials using a problem-based learning design [monografía en la Internet]. Woollongong: Australasian Society for Computers in Learning in Tertiary Education; 1998 [cited 2005 Aug 4]. Disponible en: http://www.usq.edu.au/users/albion/papers/ ascilite $98 . \mathrm{html}$

14. Willis J. Alternative instructional design paradigms: What's worth discussing and what isn't. Educational Technology. 1998;38(3):5-16.

15. Norman G, Schmidt H. The psychological basis of problembased learning: A review of the evidence. Academic Medicine. 1992;67(9):557-65.

Manuscrito recibido el 11 de mayo de 2005 y aceptado para publicación el 14 julio de 2005.

Correspondencia: Dr. Aníbal Velásquez

Av. del Sur No. 368, Dpto. 304. Chacarilla, Surco

Lima 33, Perú

Correo-e: anibal.velasquez@gmail.com 